\title{
Cytoplasm-predominant Pten associates with increased region-specific brain tyrosine hydroxylase and dopamine D2 receptors in mouse model with autistic traits
}

Xin He ${ }^{1,2}$, Stetson Thacker ${ }^{1,2,3}$, Todd Romigh ${ }^{1,2}$, Qi Yu ${ }^{1,2}$, Thomas W. Frazier $\mathrm{Jr}^{1,2,3,4,5}$ and Charis Eng ${ }^{1,2,3,6,7,8,9^{*}}$

\begin{abstract}
Background: Autism spectrum disorder (ASD) is a group of neurodevelopmental disorders characterized by impairment in social communication/interaction and inflexible/repetitive behavior. Several lines of evidence support genetic factors as a predominant cause of ASD. Among those autism susceptibility genes that have been identified, the PTEN tumor suppressor gene, initially identified as predisposing to Cowden heritable cancer syndrome, was found to be mutated in a subset of ASD patients with extreme macrocephaly. However, the ASD-relevant molecular mechanism mediating the effect of PTEN mutations remains elusive.

Methods: We developed a Pten knock-in murine model to study the effects of Pten germline mutations, specifically altering subcellular localization, in ASD. Proteins were isolated from the hemispheres of the male littermates, and Western blots were performed to determine protein expression levels of tyrosine hydroxylase $(\mathrm{TH})$. Immunohistochemical stains were carried out to validate the localization of $\mathrm{TH}$ and dopamine D2 receptors (D2R). PC12 cells ectopically expressing either wild-type or missense mutant PTEN were then compared for the differences in TH expression.

Results: Mice carrying Pten mutations have high TH and D2R in the striatum and prefrontal cortex. They also have increased phosphorylation of CAMP response element-binding protein (CREB) and TH. Mechanistically, PTEN downregulates TH production in PC12 cells via inhibiting the phosphoinositide 3-kinase (PI3K)/CREB signaling pathway, while PTEN reduces TH phosphorylation via suppressing MAPK pathway. Unlike wild-type PTEN but similar to the mouse knock-in mutant Pten, three naturally occurring missense mutations of PTEN that we previously identified in ASD patients, H93R, F241S, and D252G, were not able to suppress TH when overexpressed in PC12 cells. In addition, two other PTEN missense mutations, C124S (pan phosphatase dead) and G129E (lipid phosphatase dead), failed to suppress TH when ectopically expressed in PC12 cells.
\end{abstract}

Conclusions: Our data reveal a non-canonical PTEN-TH pathway in the brain that may work as a core regulator of dopamine signaling, which when dysfunctional is pathogenic in ASD.

Keywords: PTEN, Autism, Gene, Dopamine, Tyrosine hydroxylase

\footnotetext{
* Correspondence: engc@ccf.org

${ }^{1}$ Genomic Medicine Institute, Cleveland Clinic, 9500 Euclid Avenue, Mailstop NE-50, Cleveland, OH 44195, USA

${ }^{2}$ Lerner Research Institute, Cleveland Clinic, Cleveland, OH, USA

Full list of author information is available at the end of the article
} 


\section{Background}

Autism spectrum disorder (ASD) is a group of neurodevelopmental disorders characterized by impairments in social interaction and communication, along with the presence of inflexible/repetitive behaviors [1]. Although a strong genetic component to ASD has been confirmed by behavioral genetic studies, only 10-20\% of cases have a known genetic etiology [2].

The phosphatase and tensin homologue deleted on chromosome ten (PTEN) gene encodes a dual-specificity phosphatase that suppresses the activity of the class Ia phosphoinositide 3-kinase (PI3K) pathway and the MAPK pathway [3-5]. Cowden syndrome, an inherited breast and thyroid cancer syndrome, is associated with germline mutations of the PTEN tumor suppressor gene on 10q23 [6, 7]. Subsequently, PTEN was shown to also play an important role in brain development and plasticity [8, 9]. In Pten conditional knockout mice, Pten deficiency in brain causes dramatically weakened synaptic transmission and defects in myelination of axons [10]. We first identified germline PTEN mutations in a subset of patients with ASD and extreme macrocephaly [11], an observation that was subsequently confirmed by multiple independent groups [12-14]. An estimated $7 \%$ of children with ASD and macrocephaly carry a germline PTEN mutation [12]. Despite the macrocephaly, patients who have ASD and PTEN mutations have a cortex of normal thickness [15]. They also have an overgrowth of white matter and deficits in working memory and processing [15].

To provide insight into underlying causes for this disorder, we previously developed a germline Pten knock-in murine model of ASD. The male Pten ${ }^{\mathrm{m} 3 \mathrm{~m} 4 / \mathrm{m} 3 \mathrm{~m} 4}$ mutant mice have extreme macrocephaly due to megencephaly [16]. They also mimic individuals with high-functioning ASD, displaying increased social motivation, poor balance, and normal recognition memory [17]. It is intriguing that germline mutations of PTEN, which predispose individuals to specific cancer types, can also be linked with ASD. Unfortunately, the molecular mechanism of PTEN mutation-positive ASD (herein, PTENASD) remains unknown. Disturbed catecholamine metabolism has been reported in patients with ASD [18]. The urinary dopamine levels are reportedly significantly lower than those in normal children and are inversely correlated with the severity of autistic behavior [19]. Variants in the dopamine transporter gene $(D A T)$ and the dopamine-3-receptor gene (DRD3) are associated with ASD [20, 21]. Adult mice with conditional Pten knockout in dopaminergic neurons exhibit elevated expression of tyrosine hydroxylase, the key enzyme for dopamine synthesis [22]. Based on the fact that PTEN deletion enhances survival and function of dopamine neurons [23], we sought to address the hypothesis that PTEN works as a key player in the regulation of the dopaminergic signaling in the brain, potentially through a non-canonical signaling pathway.

\section{Methods}

Murine model study

$P_{t e n}{ }^{\mathrm{m} 3 \mathrm{~m} 4}$ missense knock-in mutant mice were generated in our lab and were backcrossed more than ten times onto a CD1 genetic background [24]. Animals were sacrificed at 8 weeks to harvest brains. The $\mathrm{m} 3 \mathrm{~m} 4$ dual Pten mutations are located in exon 7 (Fig. 1a). This exon is a hot spot for PTEN germline mutations in ASD patients (for instance, F241S, D252G). All protocols involving mice were approved by the Institutional Animal Care and Use Committee (IACUC) at the Cleveland Clinic.

\section{Reagents}

Cycloheximide was purchased from Sigma-Aldrich (St. Louis, MO). The mitogen-activated protein kinase (MAPK)/ERK kinase (MEK) inhibitor, PD98059, was purchased from Calbiochem (La Jolla, CA). The PI3K inhibitor, LY294002, was purchased from Cayman Chemical (Ann Arbor, MI). The AKT inhibitor, Perifosine (>99\% pure), was purchased from LC laboratories (Woburn, MA). PTEN phosphatase inhibitor SF1670 was purchased from Cayman Chemical (Ann Arbor, MI). Nerve growth factor (NGF 2.5S) was purchased from Life Technologies (Grand Island, NY).

\section{Cell culture}

The PC12 rat pheochromocytoma cell line was purchased from ATCC, where they authenticate by STRS analysis, and has endogenous wild-type Pten. We chose PC12 cells because it is one of the standard models for studying neural organs and, more importantly, to reflect both the ASD and neoplastic phenotypes seen in those with PTEN mutations. Mycoplasma contamination is routinely monitored. PC12 cells were grown in RPMI supplemented with $2 \mathrm{mM}$ glutamine, $5 \%$ fetal bovine serum (FBS), and $5 \%$ horse serum. Experiments were performed on cells passaged no more than ten times.

\section{Generation of stable Tet-Off cell lines}

The PC12 Tet-Off cell line was purchased from Clontech (Clontech Laboratories, Inc., Mountain View, CA, Cat\#630906). Stable cell lines with Tet-regulated PTEN expression were generated as we described previously [25]. Tet-regulated expression of exogenous PTEN was confirmed by Western blot analysis on total protein lysates collected from cells grown in the presence or absence of Tet. The wild-type (WT) construct was used as the template to engineer each of the individual mutations (TetPTEN-H93R-FLAG, TetPTENF241S-FLAG, and TetPTEN-D252G-FLAG) to be tested. Each mutant was engineered using the QuickChange in vitro site- 


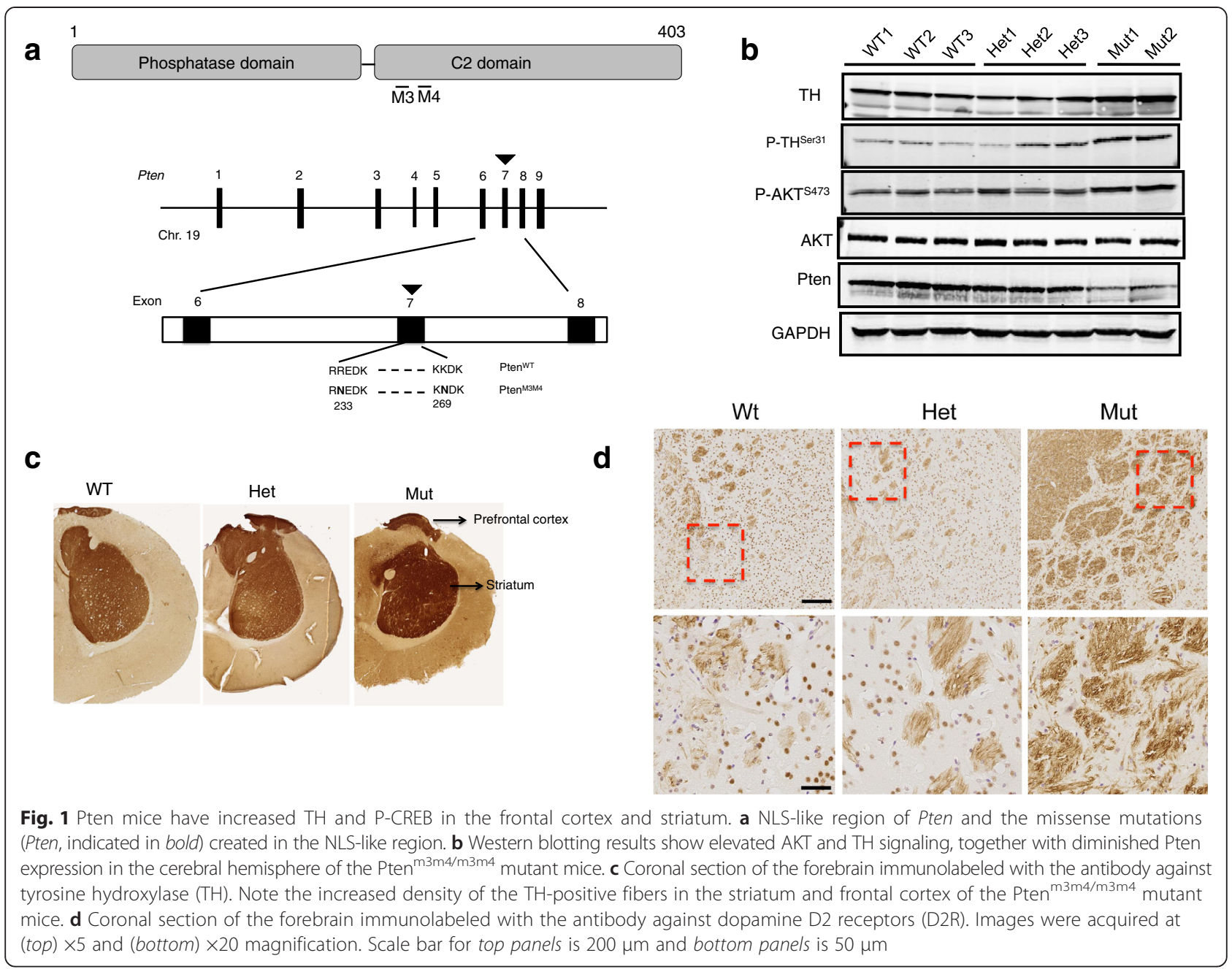

directed mutagenesis system (Stratagene) according to the manufacturer's instructions.

\section{Cycloheximide chase study}

PC12 cells stably expressing FLAG-tagged WT or mutant PTEN were incubated with cycloheximide $(50 \mu \mathrm{g} / \mathrm{mL})$ and were harvested at the indicated time points. Whole protein lysates were extracted and ran for Western blots using anti-FLAG antibody for transfected PTEN and GAPDH antibody as a loading control. Experiments were performed in triplicate.

\section{Plasmid electroporation}

CREB Dominant-Negative Vector Set (including CREB, K-CREB, CREB133) was purchased from Clontech Laboratories (Mountain View, CA). For electroporation, 4D-Nucleofector was used according to the protocol from Lonza.

\section{Western blot}

Whole cell lysates were extracted from brain tissues or PC12 cells by using RIPA buffer. Experiments were replicated in triplicate. All antibodies were purchased from Cell Signaling Technology (Beverly, MA) except tyrosine hydroxylase $(\mathrm{TH})$ antibody, which was obtained from Abcam (Cambridge, MA).

\section{Immunohistochemistry}

Briefly, mouse brains were fixed by intracardiac perfusion with $4 \%$ paraformaldehyde in phosphate-buffered saline (PBS) and postfixation in the same buffer. Specimens were then embedded in paraffin, and sections processed for immunohistochemical staining with antibody against TH (1:100) or dopamine D2 receptor (1:50, Biorbyt Ltd, Cambridge, UK). Slides were scanned at $\times 20$ using a Leica SCN400 slide scanner (Leica Microsystems Inc., Buffalo Grove, IL). Immunofluorescent staining was carried out with dopamine D2 
receptor, and the slides were imaged at $\times 20$ using a Leica DMI3000 B.

\section{Results \\ Pten ${ }^{\mathrm{m} 3 \mathrm{~m} 4}$ mutant mice have increased $\mathrm{TH}$ in the prefrontal cortex and striatum}

To address whether the $\mathrm{TH}$ and dopamine pathway in the brain plays an important role in ASD, we first examined the $\mathrm{TH}$ protein levels by Western blot in the cerebrum of male littermates aged 8 weeks (Fig. 1b). $\mathrm{TH}$ was significantly elevated in the mutant cerebrum (Fig. 1b). A similar trend of increase was also found for phospho-AKT $^{\text {Ser473 }}$ (P-AKT) and phospho-TH ${ }^{\text {Ser31 }}$ (P$\mathrm{TH})$, whereas Pten protein was significantly diminished in the mutant mice when compared to the WT littermates (Fig. 1b).

To validate the region of elevated $\mathrm{TH}$ in the $\mathrm{Pten}^{\mathrm{m} 3 \mathrm{~m} 4 /}$ m3m4 mutant mouse brains, we then performed immunohistochemical staining of $\mathrm{TH}$. Elevated $\mathrm{TH}$ was mainly distributed in the prefrontal cortex and striatum (Fig. 1c). Downstream of increased dopaminergic synthesis from $\mathrm{TH}$ is the dopamine D2 receptor (D2R), which plays critical roles in cognition. Therefore, we next measured brain D2R as a surrogate for the dopamine signaling pathway using immunohistochemistry. Consistent with the $\mathrm{TH}$ data, we found significantly increased D2R in the striatum and prefrontal cortex of the mutant mice (Fig. 1d). To further verify and quantify the increase in D2R expression in mutants, frontal sections of mouse brain were immunofluorescently stained, showing a significant increase in D2R expression in the mutant compared to WT (see Additional file 1: Figure S1). Taken together, these data suggest that the TH-dopamine-D2R pathway is upregulated in the striatum and prefrontal cortex of Pten ${ }^{\mathrm{m} 3 \mathrm{~m} 4 / \mathrm{m} 3 \mathrm{~m} 4}$ mutant mice.

\section{PTEN suppresses TH expression via inhibition of the PI3K/ CREB pathway}

The observation of increased AKT phosphorylation in the context of upregulation of the $\mathrm{TH}$ and dopamine pathways led to our hypothesis that this unexpected upregulation of the TH-dopamine pathway may be mediated by uncurbed PI3K signaling downstream of the Pten mutations. To test this hypothesis, we generated rat pheochromocytoma (PC12) cells that stably overexpress human PTEN under the control of the Tet-Off promoter, and the expression of $\mathrm{TH}$ was analyzed by Western blot. Ectopic PTEN expression was associated with decreased P-AKT and TH levels in PC12 cells (Fig. 2a, lane 2) compared to PC cells in its native state (Fig. 2a, lane 1). In this experiment, we were surprised to see that ectopic expression of human WT PTEN was associated with decreased phosphorylation of CREB (P-CREB) (Fig. 2a, right panel). Because CREB is a known regulatory target for AKT [26], we hypothesized that PI3K-dependent P-CREB allows TH transcription and hence translation.

To test our hypothesis that PI3K-dependent P-CREB facilitates $\mathrm{TH}$ transcription/translation, we sought to determine the levels of P-AKT, P-CREB, and TH after inhibition of the PI3K pathway as well as after stimulation of the PI3K pathway. If our hypothesis is correct, then inhibition of PI3K should result in the opposite of our above observation, i.e., decreased expression of TH, P$\mathrm{AKT}$, and P-CREB. We achieved PI3K inhibition via two different, and standard, approaches: pharmacologic inhibition of PI3K by LY294002 and serum starvation. First, we treated PC12 cells overnight with LY294002 (Fig. 2b, left panel, +). As a PI3K inhibitor, LY294002 was expected, and is observed, to decrease P-AKT expression (while AKT expression with and without treatment were identical). Notably, LY294002 also resulted in decreased TH and decreased P-CREB (CREB expression with and without treatment were similar) (Fig. 2b, left panel, + vs -). Similarly, serum starvation decreased $\mathrm{P}-\mathrm{AKT}$, as well as P-CREB, and TH (Fig. 2b, middle panel). We then chose NGF as our upregulator of PI3K and MAPK as an unrelated mimic of dysfunctioning/nonfunctioning PTEN: if our hypothesis remains correct, then NGF should be seen to increase P-AKT, P-CREB, and TH. When we exposed PC12 cells to NGF, Western blot indeed revealed increased expression of P-AKT, P-CREB, and $\mathrm{TH}$ (Fig. 2b, right panel). Thus, we were able to confirm that the PI3K pathway is important in mediating P-CREB, leading to transcriptional control of $\mathrm{TH}$ expression, at least in vitro.

To test whether CREB phosphorylation is sufficient to facilitate $\mathrm{TH}$ expression, we transfected PC12 cells with $\mathrm{CREB}^{\mathrm{WT}}$ and two different CREB mutant constructs, $\mathrm{CREB}^{\mathrm{S133A}}$ and K-CREB (Fig. 2c). $\mathrm{CREB}^{\mathrm{S133A}}$ is a mutant in which serine 133, the major phosphorylation site of CREB, is changed to alanine, thus abrogating its ability to be phosphorylated. If our hypothesis is correct, then $\mathrm{CREB}^{\mathrm{S} 133 \mathrm{~A}}$ should be associated with no/little P-CREB and decreased TH. Expression of the mutant CREB ${ }^{\text {S133A }}$ is, in fact, associated with dramatically decreased P-CREB, as expected; of note, $\mathrm{CREB}^{\mathrm{S} 133 \mathrm{~A}}$ transfection is also associated with decreased $\mathrm{TH}$ (Fig. 2c, right lane), thus confirming our hypothesis. K-CREB is a dominantnegative mutant form of CREB. If our hypothesis is correct, K-CREB should decrease TH, without affecting P-CREB. Indeed, expression of K-CREB decreased $\mathrm{TH}$ without markedly reducing the P-CREB (Fig. 2c, middle lane). Of relevance, we identified, via bioinformatic analysis, a CREB consensus binding site at the 5'UTR of the TH gene (Fig. 2d). As partial in vivo validation of our observations in PC12 cells, we were able to show elevated CREB phosphorylation in the cerebrum of the Pten ${ }^{\mathrm{m} 3 \mathrm{~m} 4 / \mathrm{m} 3 \mathrm{~m} 4}$ mice (Fig. 2e) where 


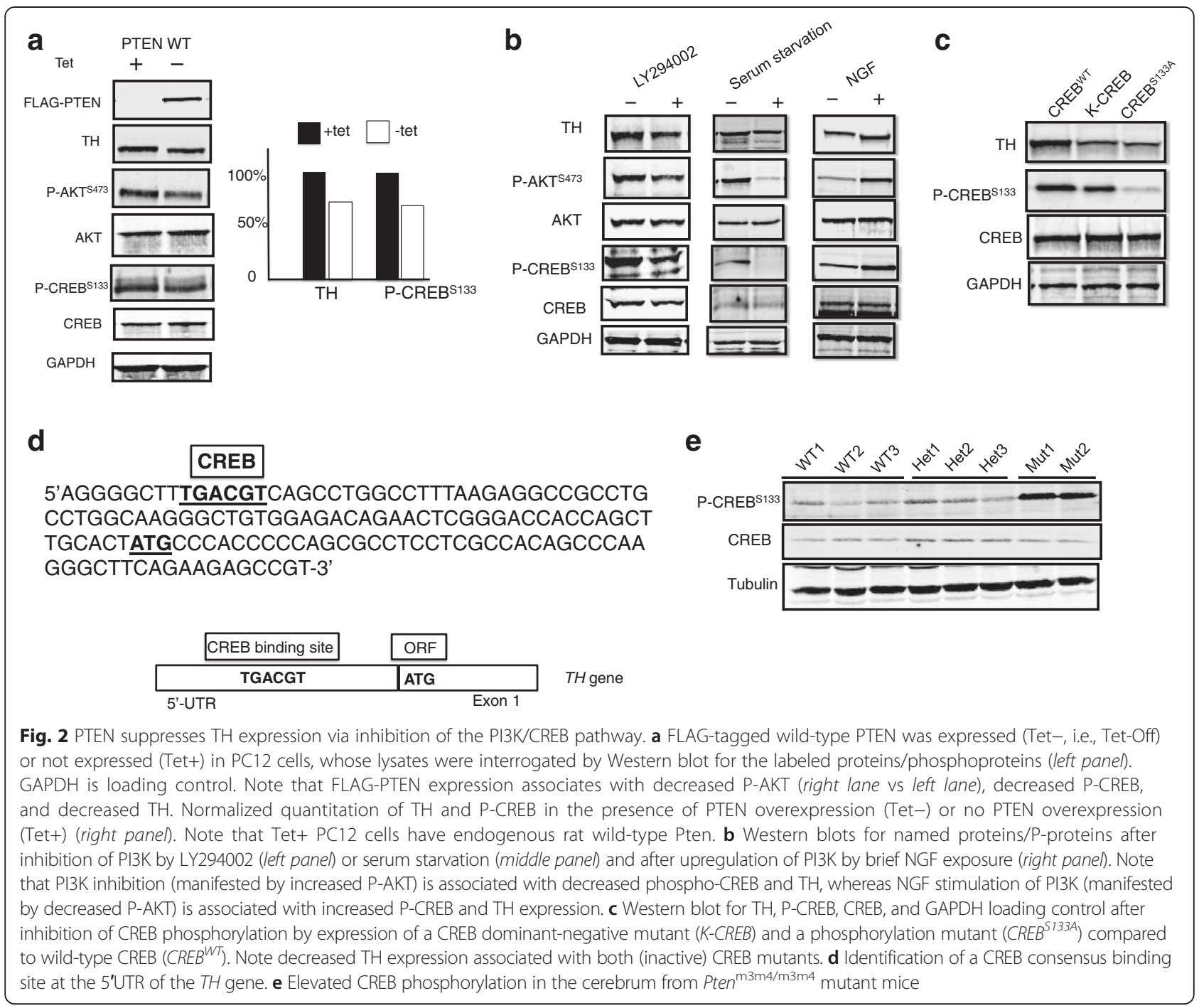

we have already shown increased TH expression (Fig. 1c). Taken together, our observations suggest that mutant PTEN is associated with upregulation of the PI3K pathway, manifested by increased P-AKT, resulting in CREB phosphorylation and subsequent increased $\mathrm{TH}$ transcription. Said another way, functioning (WT) PTEN suppresses TH expression via inhibition of the PI3K/ CREB pathway.

\section{Wild-type PTEN reduces the phosphorylation of TH}

We have already observed increased $\mathrm{TH}$ and increased phosphorylated TH in the cerebrum of our Pten mutant mice (above, Fig. 1b). Phosphorylation of TH is already known to increase $\mathrm{TH}$ protein stability and activity $[27,28]$. Thus, we sought to determine if PTEN can also regulate $\mathrm{TH}$ phosphorylation.

PC12 cells at rest have low levels of P-TH (Fig. 3a, left panel, lane 1). We used NGF to make PC12 cells active; with only 30 min of NGF treatment, P-TH was significantly elevated (Fig. 3a, left panel, lane 3). The overexpression of PTEN (Tet-, i.e., Tet-Off turns on transfected PTEN) caused decreased P-TH (Fig. 3a, left panel, lane 4 [Tet-, PTEN on] vs lane 2 [Tet+, PTEN off]). After densitometry normalization, we found that total $\mathrm{TH}$ decreased $\sim 20 \%$ after exogenous PTEN expression, whereas the P-TH decreased $\sim 50 \%$ after exogenous PTEN expression (Fig. 3a, right panel). To further illustrate the effect of PTEN on TH phosphorylation, we used a PTEN inhibitor called SF1670 to inhibit both the ectopically expressed PTEN and the endogenously expressed PTEN in our PC12 cell line. A dramatic increase in phosphorylation at $\mathrm{S} 31$ on TH in PC12 cells was observed after treating for $3 \mathrm{~h}$ with the $\mathrm{IC}_{50}$ of SF1670. Moreover, a modest increase was observed in S40 phosphorylation. Consistent with our immunostaining of the $\mathrm{m} 3 \mathrm{~m} 4$ mouse brains, an increase in D2R expression was also observed after inhibition of PTEN with SF1670 (see Additional file 1: Figure S2). Therefore, these observations 

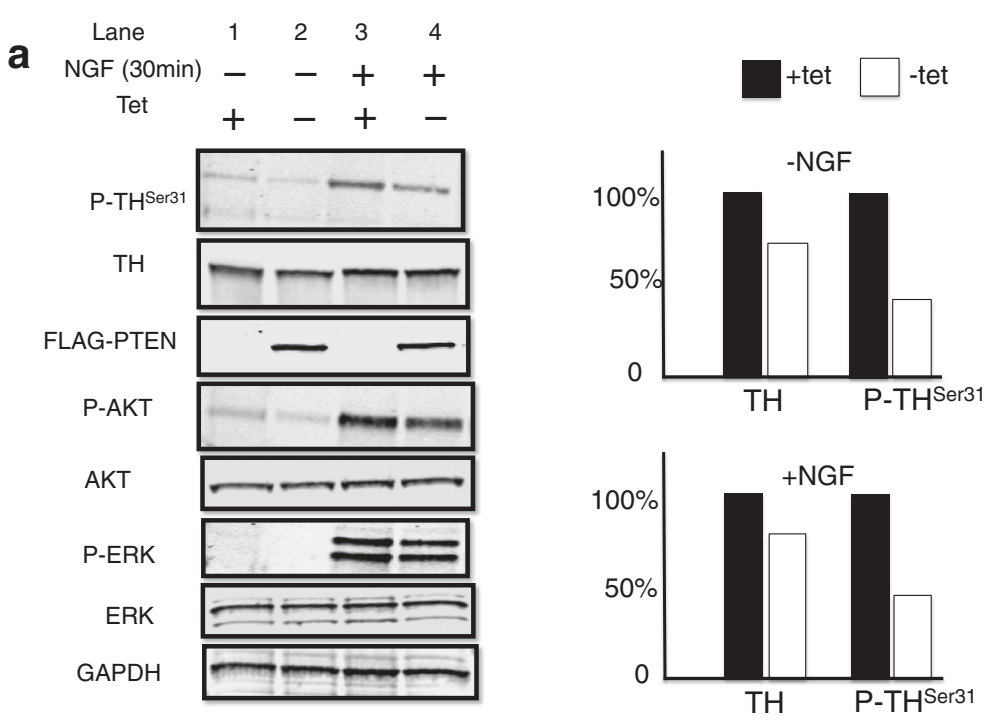

b

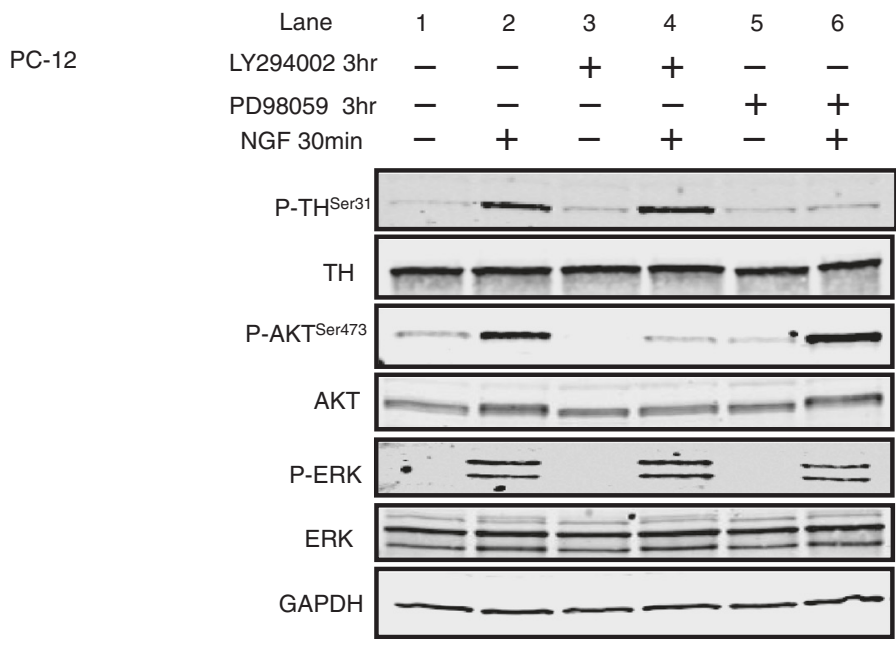

Fig. 3 PTEN suppresses TH phosphorylation and stability via inhibition of the ERK/MAPK pathway in PC12 cells. a Western blot of the named proteins/phosphoproteins after exposure of NGF (to activate PC12 cells from resting state) or not, in the presence (Tet-) or absence (Tet+) of exogenous PTEN expression (left panel). Note that expression of PTEN reduces TH phosphorylation in PC12 cells. Normalized quantitation of relative TH and P-TH expression in the presence (white bar, Tet-) or absence (black bar, Tet+) of exogenous PTEN expression with (NGF+) or without (NGF-) exposure (right bar graphs). b Western blot of the named proteins/phosphoproteins in the presence (+) or absence (-) of MAPK inhibitor PD98059 or of PI3K inhibitor LY294002 with (+) or without (-) NGF stimulation of PC12 cells. Note that MAPK inhibitor PD98059, but not PI3K inhibitor LY294002, is associated with decreased P-TH even after NGF stimulation

suggest that normal PTEN signaling inhibits TH expression (see above sections) as well as phosphorylation of $\mathrm{TH}$.

PTEN can act as both a protein and lipid phosphatase that inhibits both MAPK and PI3K pathways. We have already shown (above sections) that PTEN inhibits the PI3K pathway to decrease CREB phosphorylation and total TH levels. Now, we would also like to determine if inhibition of MAPK and/or PI3K would affect TH phosphorylation. To minimize the effect of the change on total $\mathrm{TH}$ by chemical inhibitors, we only treated cells for
3 h with either the PI3K inhibitor, LY294002, or the MAPK inhibitor, PD98059, in media without serum. After 3 h, PC12 cells were treated with NGF for $30 \mathrm{~min}$ to awaken resting $\mathrm{PC} 12$ cells. NGF-stimulated $\mathrm{PC} 12$ cells had increased phosphorylation of TH without altering the level of total TH (Fig. 3b, lane 2). Surprisingly, PD98059, but not LY294002, inhibited NGF-stimulated TH phosphorylation in PC12 cells (Fig. 3b, lane 4 vs 6). Therefore, PTEN appears to suppress the phosphorylation of $\mathrm{TH}$ through inhibition of MAPK, probably through its protein phosphatase activity. Corroborating 
this, functional PTEN inhibitor SF1670, even in the presence of PTEN overexpression, resulted in increased P-ERK, slightly increased $\mathrm{TH}$, and clearly increased P-TH (see Additional file 1: Figure S2 comprising lane 3 vs lane 2).

\section{Three PTEN missense mutants identified in ASD patients cannot suppress $\mathrm{TH}$}

In order to validate the role of PTEN mutations in ASD patients, we transfected three naturally occurring ASD-associated PTEN mutations into PC12 cells to see if these mutant PTEN constructs can suppress $\mathrm{TH}$ in vitro. If our mouse and $\mathrm{PC} 12$ data above are correct, then these three ASD-associated PTEN germline mutations should not be able to suppress TH levels. Indeed, all three mutations, H93R, F241S, and D252G, cannot suppress $\mathrm{TH}$ when expressed in PC12 cells. They also failed to suppress P-AKT (Fig. 4a, left panel). We then investigated $\mathrm{TH}$ expression after expression of PTEN mutations, C124S which is pan phosphatase dead and G129E which is only lipid phosphatase dead. Next, we investigated $\mathrm{TH}$ phosphorylation when these mutants were expressed in the presence of PTEN inhibitor SF1670.
The three different PTEN mutations varied in their ability to suppress $\mathrm{TH}$ phosphorylation at S31 and S40; however, the general trend was an inability to suppress phosphorylation of TH as effectively as WT PTEN (see Additional file 1: Figure S3). Expression of both PTEN mutants in PC12 cells could not suppress P-AKT, TH, or P-TH (Fig. 4a, right panel). Again, our results underscore that the intact lipid phosphatase activity is necessary for PTEN to decrease TH expression, while intact protein phosphatase activity is necessary for PTEN to inhibit $\mathrm{TH}$ phosphorylation.

\section{Diminished protein stability in ASD-associated PTEN missense mutations}

Based on our observations that the $\operatorname{Pten}^{\mathrm{m} 3 \mathrm{~m} 4 / \mathrm{m} 3 \mathrm{~m} 4}$ mutant mice have diminished Pten protein levels in the brain [17], we hypothesized that ASD-associated PTEN mutations decrease the stability of PTEN protein. We therefore interrogated PTEN protein stability through a cycloheximide chase study (Fig. 4b). The WT PTEN protein was stable after synthesis and we observed that its levels remain at $\sim 100 \%$ even after $8 \mathrm{~h}$ of cycloheximide treatment.

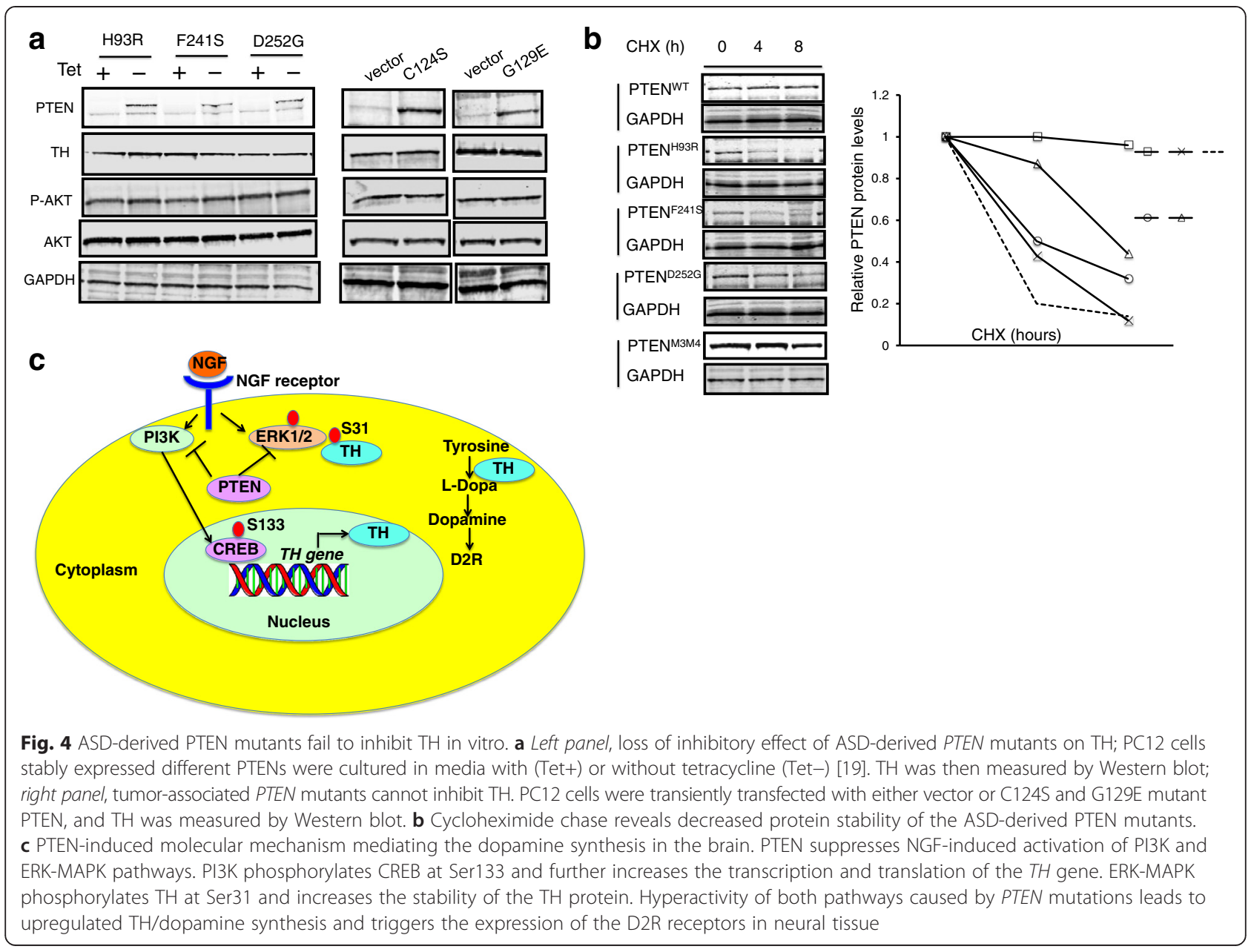


In contrast, H93R, F241S, and D252G and m3m4 PTEN/ Pten mutant proteins are highly unstable. More than $50 \%$ of the mutant $\mathrm{m} 3 \mathrm{~m} 4$ and D252G PTEN proteins were degraded after $8 \mathrm{~h}$ of cycloheximide treatment. For H93R and F241S, more than $80 \%$ of the mutant PTEN protein was degraded after $8 \mathrm{~h}$ of cycloheximide treatment (Fig. 4b).

\section{Discussion}

The present study demonstrates the increased expression and phosphorylation of $\mathrm{TH}$ in the prefrontal cortex and striatum of a Pten knock-in ASD mouse model. Our data indicate that functioning PTEN suppresses $T H$ transcription through the inhibitory effect on PI3K and P-CREB, whereas PTEN also inhibits TH phosphorylation through suppressing the MAPK pathway (Fig. 4c). Thus, PTEN inhibits TH-dopamine pathway through its role as dual protein and lipid phosphatase.

Dopamine plays a key role in the regulation of locomotion, learning, behavior, emotion, and social interaction. $\mathrm{TH}$ is the rate-limiting enzyme in the synthesis of dopamine. Therefore, it is reasonable to posit that dysregulation of the dopaminergic system is linked to the pathophysiology of ASD. Of relevance, we have shown that mutations of the PTEN gene augment TH synthesis and function, and this may lead to the release of too much dopamine in the prefrontal cortex and midbrain, which can result in repetitive and obsessive behaviors. This can also explain the motor disorders (such as poor balance) reported in our mouse model [17]. Intriguingly, several groups have reported dysregulated dopamine function in ASD patients. Cartier et al. reported that two rare variants in STX1A and SLC6A3 that associate with autism disrupt reverse transport of dopamine [29]. This same group also found that another variant of SLC6A3, the dopamine transporter gene, caused alterations in dopamine transport function and trafficking, which associated with ASD in two different families [30]. A Japanese group found that dopamine transporter binding was significantly higher in the orbitofrontal cortex of autistic individuals [31]. Furthermore, dysfunction of Pten has been associated with potential disruption of dopamine function and ASD behaviors in other mouse models. Conditional knockout of Pten in dopaminergic neurons resulted in neuronal hypertrophy, an increased number of dopaminergic neurons and fibers in the ventral mesencephalon, and social behavioral impairments [32, 33]. It has also been demonstrated that a germline Pten haploinsufficient model has altered social behavior, mirroring the social deficits of ASD, and displays neuronal overgrowth driven by $\beta$-catenin signaling $[34,35]$. If the activated TH-dopamine pathway is germane to PTEN-ASD, there is a possibility that these symptoms could be reversed with pharmaceuticals that target the PTEN-TH-dopamine pathway.
The D2R represents the main autoreceptor of the dopaminergic system in the brain. The upregulation of striatal D2R expression is a notable finding in our study. A role for D2R in ASD susceptibility is suggested by the fact that risperidone, which prevents D2R activation, can reduce disruptive behavior in about half of the children with ASD [36]. In humans, D2R is encoded by the $D R D 2$ gene, which is a language-related gene. Variants in the DRD2 gene, especially the rs1800498TT genotype and the A1 allele of the Taq I polymorphism, have been found to influence language traits in children with ASD [37-39]. Further studies of the effects of D2R on ASD will be required in order to assess whether common mechanisms are shared with what are reported herein for PTEN-mediated inhibitory effects on the dopamine pathway.

As a dual lipid and protein phosphatase, PTEN can suppress both the PI3K and the MAPK signaling pathways. We show that PTEN inhibits TH transcription by suppressing the PI3K and CREB pathways. We also show that PTEN can also suppress TH phosphorylation through its protein phosphatase activity by inhibiting ERK-MAPK. Thus, both lipid and protein phosphatase activities of PTEN are involved in TH regulation. The ASD-associated mutations of PTEN may present a "double whammy" in leading to ASD. Not only does PTEN signaling lessen total TH but it also reduces the phosphorylation of $\mathrm{TH}$ that leads to decreased activity and stability of $\mathrm{TH}$ protein. By using three ASD-associated PTEN missense mutations (H93R, F241S, D252G), we demonstrate the loss of PTEN's inhibitory effects on P-AKT and TH and show that the lipid phosphatase activity of PTEN plays a major role in $\mathrm{TH}$ inhibition. Indeed, $T H$ gene transcription is tightly controlled by the PTEN-PI3K pathway, as reported previously [40].

\section{Conclusions}

Our observations reveal a novel inhibitory role of PTEN in the regulation of dopamine signaling via the noncanonical PTEN-TH-dopamine pathway in neural tissue. Mutations of the PTEN gene can enhance TH in two ways: the loss of the lipid phosphatase activity of PTEN will induce $\mathrm{TH}$ transcription and translation through the dysregulated PI3K/CREB pathway and the loss of the protein phosphatase activity of PTEN will enhance the phosphorylation of $\mathrm{TH}$ through the MAPK pathway. Continued investigation of the PTEN-TH-dopamine pathway in ASD will uncover novel drug targets for exploitation by new therapeutic interventions.

\section{Availability of supporting data}

Supplementary information is available at Molecular Psychiatry's website. 


\section{Additional files}

Additional file 1: Supplementary Information. Three supplementary figures comprising Figure S1A. Immunofluorescent staining of frontal sections of mouse brains indicates increased D2R expression and larger nuclei in Pten ${ }^{\mathrm{m} 3 \mathrm{~m} 4 / \mathrm{m} 3 \mathrm{~m} 4}$ mice. Figure S1B. Quantification of D2R immunofluorescence in triplicates of each genotype's frontal section of mouse brains indicates the mutant D2R immunofluorescence is significantly increased in comparison to the immunofluorescence in the wild-type $(P=0.021)$. Figure $\mathbf{S}$. Inhibition of ectopic and endogenous PTEN function with SF1670 leads to an increase in phosphorylation of TH in PC12 cells. PTEN inhibitor is applied in lanes 1 and $3(+)$ but off in lane $2(-)$. Tetracycline is on (+) in lane 1, turning off ectopic expression of wild-type PTEN in PC12 cells that has endogenous wild-type PTEN. Tet-Off (-) turns on ectopic expression of wild-type human PTEN (lanes 2 and 3). Figure S3. Overexpression of naturally occurring ASD-associated germline PTEN mutations are unable to completely suppress $\mathrm{TH}$ phosphorylation. When tetracycline is applied (Tet+), ectopic PTEN expression is turned off. When tetracycline is removed (Tet-Off; -), ectopic expression of PTEN occurs. (PDF $965 \mathrm{~kb})$

\section{Abbreviations}

ASD: autism spectrum disorder; CREB: CAMP response element-binding protein; D2R: dopamine D2 receptors; MAPK: mitogen-activated protein kinase; PHTS: PTEN hamartoma tumor syndrome; PI3K: phosphoinositide 3-kinase; TH: tyrosine hydroxylase.

\section{Competing interests}

The authors declare that they have no competing interests.

\section{Authors' contributions}

$\mathrm{XH}$ and $\mathrm{CE}$ conceived of the study, performed the data acquisition and analysis, and drafted and revised the manuscript. ST, TR, and QY participated in the acquisition of data and analysis of data and helped to critically revise the manuscript. TWF participated in the design of the study and helped to perform data analysis and to draft the manuscript. All authors read and approved the final manuscript.

\section{Acknowledgements}

The authors wish to thank Hugh Hiu Nam Chan and Blake Chafee for helpful discussions and technical guidance for the dopamine receptor studies. We are indebted to Jessica Mester and Kimberly Stanuch for their databasing assistance. The authors are also grateful to the patients and their parents who participated in this study. This work was supported, in part, by the National Institutes of Health (R01CA118989 to CE; U54NS092090 to CE and TWF) and a generous gift from Sam H Miller. CE is the Sondra J and Stephen R Hardis Endowed Chair of Cancer Genomic Medicine at the Cleveland Clinic and is an ACS Clinical Research Professor.

\section{Author details}

${ }^{1}$ Genomic Medicine Institute, Cleveland Clinic, 9500 Euclid Avenue, Mailstop NE-50, Cleveland, OH 44195, USA. 'Lerner Research Institute, Cleveland Clinic, Cleveland, $\mathrm{OH}, \mathrm{USA} .{ }^{3} \mathrm{HHMI}$ Graduate Program, Department of Molecular Medicine, Cleveland Clinic Lerner College of Medicine, Case Western Reserve University School of Medicine, Cleveland, OH, USA. ${ }^{4}$ Center for Autism, Pediatrics Institute, Cleveland Clinic, Cleveland, OH, USA. ${ }^{5}$ Department of Pediatrics, Case Western Reserve University School of Medicine, Cleveland, $\mathrm{OH}$, USA. ${ }^{6}$ Taussig Cancer Institute, Cleveland Clinic, Cleveland, OH, USA. ${ }^{7}$ Stanley Shalom Zielony Institute of Nursing Excellence, Cleveland Clinic, Cleveland, OH, USA. ${ }^{8}$ Department of Genetics and Genome Sciences, Case Western Reserve University School of Medicine, Cleveland, OH, USA. ${ }^{9}$ CASE Comprehensive Cancer Center, Case Western Reserve University School of Medicine, Cleveland, $\mathrm{OH}$, USA.

\section{Received: 8 June 2015 Accepted: 9 November 2015} Published online: 17 November 2015

\section{References}

1. American Psychiatric Association. Diagnostic and Statistical Manual of Mental Disorders, Fifth ed. Arlington: American Psychiatric Association; 2013.
2. De Rubeis S, JD Buxbaum. Genetics and genomics of autism spectrum disorder: Embracing complexity. Hum Mol Genet. 2015;24(17): e-pub ahead of print 17 July 2015; doi: 10.1093/hmg/ddv273

3. Nakdimon I, Walser M, Frohli E, Hajnal A. PTEN negatively regulates MAPK signaling during Caenorhabditis elegans vulval development. PLoS Genet. 2012;8(8):e1002881. doi:10.1371/journal.pgen.1002881.

4. Weng LP, Smith WM, Brown JL, Eng C. PTEN inhibits insulin-stimulated MEK MAPK activation and cell growth by blocking IRS-1 phosphorylation and IRS-1/Grb-2/Sos complex formation in a breast cancer model. Hum Mol Genet. 2001;10(6):605-16.

5. Sun H, Lesche R, Li DM, Liliental J, Zhang H, Gao J, et al. PTEN modulates cell cycle progression and cell survival by regulating phosphatidylinositol 3,4,5,-trisphosphate and Akt/protein kinase B signaling pathway. Proc Natl Acad Sci U S A. 1999;96(11):6199-204.

6. Marsh DJ, Dahia PL, Caron S, Kum JB, Frayling IM, Tomlinson IP, et al. Germline PTEN mutations in Cowden syndrome-like families. J Med Genet. 1998;35(11):881-5.

7. Liaw D, Marsh DJ, Li J, Dahia PL, Wang SI, Zheng Z, et al. Germline mutations of the PTEN gene in Cowden disease, an inherited breast and thyroid cancer syndrome. Nat Genet. 1997;16(1):64-7. doi:10.1038/ng0597-64.

8. Qu Q, Shi Y. Neural stem cells in the developing and adult brains. J Cell Physiol. 2009;221(1):5-9. doi:10.1002/jcp.21862.

9. Veleva-Rotse BO, Barnes AP. Brain patterning perturbations following PTEN loss. Front Mol Neurosci. 2014;7:35. doi:10.3389/fnmol.2014.00035.

10. Fraser MM, Bayazitov IT, Zakharenko SS, Baker SJ. Phosphatase and tensin homolog, deleted on chromosome 10 deficiency in brain causes defects in synaptic structure, transmission and plasticity, and myelination abnormalities. Neuroscience. 2008;151(2):476-88. doi:10.1016/j.neuroscience. 2007.10.048.

11. Butler MG, Dasouki MJ, Zhou XP, Talebizadeh Z, Brown M, Takahashi TN, et al. Subset of individuals with autism spectrum disorders and extreme macrocephaly associated with germline PTEN tumour suppressor gene mutations. J Med Genet. 2005;42(4):318-21. doi:10.1136/jmg.2004.024646.

12. McBride KL, Varga EA, Pastore MT, Prior TW, Manickam K, Atkin JF, et al. Confirmation study of PTEN mutations among individuals with autism or developmental delays/mental retardation and macrocephaly. Autism Res. 2010;3(3):137-41. doi:10.1002/aur.132.

13. Marchese M, Conti V, Valvo G, Moro F, Muratori F, Tancredi R, et al. Autism-epilepsy phenotype with macrocephaly suggests PTEN, but not GLIALCAM, genetic screening. BMC Med Genet. 2014;15:26. doi:10.1186/ 1471-2350-15-26.

14. Busa T, Milh M, Degardin N, Girard N, Sigaudy S, Longy M, et al. Clinical presentation of PTEN mutations in childhood in the absence of family history of Cowden syndrome. Eur J Paediatr Neurol. 2015;19(2):188-92. doi: 10.1016/j.ejpn.2014.11.012.

15. Frazier TW, Embacher R, Tilot AK, Koenig K, Mester J, Eng C. Molecular and phenotypic abnormalities in individuals with germline heterozygous PTEN mutations and autism. Mol Psychiatry. 2014. doi:10.1038/mp.2014.125.

16. Mester $\mathrm{L}$, Tilot AK, Rybicki LA, Frazier TW, Eng C. Analysis of prevalence and degree of macrocephaly in patients with germline PTEN mutations and of brain weight in Pten knock-in murine model. Eur J Hum Genet. 2011;19(7): 763-8. doi:10.1038/ejhg.2011.20.

17. Tilot AK, Gaugler MK, Yu Q, Romigh T, Yu W, Miller RH, et al. Germline disruption of Pten localization causes enhanced sex-dependent social motivation and increased glial production. Hum Mol Genet. 2014;23(12): 3212-27. doi:10.1093/hmg/ddu031.

18. Launay JM, Bursztejn C, Ferrari P, Dreux C, Braconnier A, Zarifian E, et al. Catecholamines metabolism in infantile autism: a controlled study of 22 autistic children. J Autism Dev Disord. 1987;17(3):333-47.

19. Barthelemy C, Bruneau N, Cottet-Eymard JM, Domenech-Jouve J, Garreau B, Lelord G, et al. Urinary free and conjugated catecholamines and metabolites in autistic children. J Autism Dev Disord. 1988;18(4):583-91.

20. Hamilton PJ, Campbell NG, Sharma S, Erreger K, Herborg Hansen F, Saunders $\mathrm{C}$, et al. De novo mutation in the dopamine transporter gene associates dopamine dysfunction with autism spectrum disorder. Mol Psychiatry. 2013;18(12):1315-23. doi:10.1038/mp.2013.102.

21. Staal WG, de Krom M, de Jonge MV. Brief report: the dopamine-3-receptor gene (DRD3) is associated with specific repetitive behavior in autism spectrum disorder (ASD). J Autism Dev Disord. 2012;42(5):885-8. doi:10.1007/s10803-011-1312-z.

22. Domanskyi A, Geissler C, Vinnikov IA, Alter H, Schober A, Vogt MA, et al. Pten ablation in adult dopaminergic neurons is neuroprotective in 
Parkinson's disease models. FASEB J. 2011;25(9):2898-910. doi:10.1096/fj. 11-181958.

23. Zhang Y, Granholm AC, Huh K, Shan L, Diaz-Ruiz O, Malik N, et al. PTEN deletion enhances survival, neurite outgrowth and function of dopamine neuron grafts to MitoPark mice. Brain. 2012;135(Pt 9):2736-49. doi:10.1093/ brain/aws196.

24. Mester JL, Tilot AK, Rybicki LA, Frazier 2nd TW, Eng C. Analysis of prevalence and degree of macrocephaly in patients with germline PTEN mutations and of brain weight in Pten knock-in murine model. Eur J Hum Genet. 2011; 19(7):763-8. doi:10.1038/ejhg.2011.20.

25. Lobo GP, Waite KA, Planchon SM, Romigh T, Nassif NT, Eng C. Germline and somatic cancer-associated mutations in the ATP-binding motifs of PTEN influence its subcellular localization and tumor suppressive function. Hum Mol Genet. 2009;18(15):2851-62. doi:10.1093/hmg/ddp220.

26. Du K, Montminy M. CREB is a regulatory target for the protein kinase Akt/ PKB. J Biol Chem. 1998;273(49):32377-9.

27. Dunkley PR, Bobrovskaya L, Graham ME, von Nagy-Felsobuki El, Dickson PW. Tyrosine hydroxylase phosphorylation: regulation and consequences. Journal of Neurochemistry. 2004;91(5):1025-43. doi:10.1111/j.1471-4159.2004.02797.x.

28. Moy LY, Tsai LH. Cyclin-dependent kinase 5 phosphorylates serine 31 of tyrosine hydroxylase and regulates its stability. J Biol Chem. 2004;279(52): 54487-93. doi:10.1074/jbc.M406636200.

29. Cartier E, Hamilton PJ, Belovich AN, Shekar A, Campbell NG, Saunders C, et al. Rare autism-associated variants implicate syntaxin 1 (STX1 R26Q) phosphorylation and the dopamine transporter (hDAT R51W) in dopamine neurotransmission behaviors. EBioMedicine. 2015;2(2):135-46. doi:10.1016/j. ebiom.2015.01.007.

30. Bowton E, Saunders C, Reddy IA, Campbell NG, Hamilton PJ, Henry LK, et al. SLC6A3 coding variant Ala559Val found in two autism probands alters dopamine transporter function and trafficking. Transl Psychiatry. 2014;14(4): e464. doi:10.1038/tp.2014.90.

31. Nakamura K, Sekine Y, Ouchi Y, Tsujii M, Yoshikawa E, Futatsubashi M, et al. Brain serotonin and dopamine transporter bindings in adults with highfunctioning autism. Arch Gen Psychiatry. 2010;67(1):59-68. doi:10.1001/ archgenpsychiatry.2009.137.

32. Diaz-Ruiz O, Zapata A, Shan L, Zhang Y, Tomac AC, Malik N, et al. Selective deletion of PTEN in dopamine neurons leads to trophic effects and adaptation of striatal medium spiny projecting neurons. PLoS One. 2009; 4(9):e7027. doi:10.1371/journal.pone.0007027.

33. Clipperton-Allen AE, Page DT. Pten haploinsufficient mice show broad brain overgrowth but selective impairments in autism-relevant behavioral tests. Hum Mol Genet. 2014;23(13):3490-505. doi:10.1093/hmg/ddu057.

34. Clipperton-Allen AE, Page DT. Decreased aggression and increased repetitive behavior in Pten haploinsufficient mice. Genes Brain Behav. 2015; 14(2):145-57. doi:10.1111/gbb.12192

35. Chen Y, Huang WC, Séjourné J, Clipperton-Allen AE, Page DT. Pten mutations alter brain growth trajectory and allocation of cell types through elevated $\beta$-catenin signaling. J Neurosci. 2015;35(28):10252-67. doi:10.1523/JNEUROSCI.5272-14.2015.

36. Troost PW, Lahuis BE, Steenhuis MP, Ketelaars CE, Buitelaar JK, van Engeland $\mathrm{H}$, et al. Long-term effects of risperidone in children with autism spectrum disorders: a placebo discontinuation study. J Am Acad Child Adolesc Psychiatry. 2005;44(11):1137-44. doi:10.1097/01.chi.0000177055.11229.76.

37. Hettinger JA, Liu X, Hudson ML, Lee A, Cohen IL, Michaelis RC, et al. DRD2 and PPP1R1B (DARPP-32) polymorphisms independently confer increased risk for autism spectrum disorders and additively predict affected status in male-only affected sib-pair families. Behav Brain Funct. 2012:8:19. doi:10. 1186/1744-9081-8-19.

38. Eicher JD, Gruen JR. Language impairment and dyslexia genes influence language skills in children with autism spectrum disorders. Autism Res. 2014. doi:10.1002/aur.1436.

39. Comings DE, Comings BG, Muhleman D, Dietz G, Shahbahrami B, Tast D, et al. The dopamine $D 2$ receptor locus as a modifying gene in neuropsychiatric disorders. JAMA. 1991;266(13):1793-800.

40. Musatov S, Roberts J, Brooks Al, Pena J, Betchen S, Pfaff DW, et al. Inhibition of neuronal phenotype by PTEN in PC12 cells. Proc Natl Acad Sci U S A. 2004;101(10):3627-31. doi:10.1073/pnas.0308289101.

\section{Submit your next manuscript to BioMed Central and take full advantage of:}

- Convenient online submission

- Thorough peer review

- No space constraints or color figure charges

- Immediate publication on acceptance

- Inclusion in PubMed, CAS, Scopus and Google Scholar

- Research which is freely available for redistribution 